\title{
Modeling Signal-Determining Radiation Components of Microbolometer-Based Infrared Measurement Systems
}

\author{
A. Tempelhahn, H. Budzier, V. Krause, G. Gerlach \\ Technische Universität Dresden \\ Solid-State Electronics Laboratory \\ 01062 Dresden \\ Germany \\ Alexander.Tempelhahn@tu-dresden.de
}

\begin{abstract}
:
This paper studies influences of changing external temperature conditions on radiation measurement using microbolometer-based infrared focal plane arrays (FPA). The effect of thermal drift on the radiation measurement using TEC-less infrared cameras is compared to the effect on measurements with TEC. Sensor specific parameters are analyzed and their correlations due to variations in temperature are described. Using a simplified radiation measurement set-up the influence of a defined temperature gradient on the measured radiation signal is analyzed and compared with the calculated radiation responds.
\end{abstract}

Key words: microbolometer FPA, TEC-less, thermal drift

\section{Introduction}

Infrared detectors are divided into two groups, photon and thermal infrared sensors. Thermal infrared sensors are sensitive over a wide spectral band. They do not need to be cooled like most of photon sensors, what makes them cheap and comparatively small. Therefore, thermal infrared sensors, especially microbolometer senor 2D-arrays (focal plane arrays), are highly suitable for low-cost applications like thermovision and radiometric thermography [1]. This paper considers the modeling of the signal-determining radiation components as prerequisite for radiometric design of sensor arrays. For that reason, it will focus on this kind of infrared detectors, but conclusions may be adaptable for other detector types.

To achieve small measurement uncertainty values for radiometric infrared cameras one has to deal with several problems like changing external conditions (e.g. ambient temperature) and their effect on sensor specific parameters. Main goal of this paper is a separate analyze of the specific camera and sensor parameters and their behavior according to variations of ambient conditions, especially changes of ambient temperature. Combining all influences should allow to estimate the final measurement uncertainty and to introduce particular measures to minimize it.

\section{Thermal drift experiments}

The following study will focus on ULIS infrared sensors with a resolution of $640 \times 480$ pixels which are used in the 8-14 $\mu \mathrm{m}$ spectral band. The sensor temperature can be stabilized using a thermo electric cooler (TEC). In a first experiments two cameras are compared, one with a TEC-controlled constant sensor temperature at $30{ }^{\circ} \mathrm{C}$ and a second one without TEC, hence, steadily increasing temperature (thermal drift). Table 1 compares both sensor types used here.

Tab. 1: Key properties of both used sensor types.

\begin{tabular}{|c|c|c|}
\hline sensor type & $\begin{array}{c}\text { UL04171- } \\
011\end{array}$ & $\begin{array}{c}\text { UL04272- } \\
032\end{array}$ \\
\hline $\begin{array}{c}\text { integrated } \\
\text { TEC }\end{array}$ & yes & no \\
\hline NETD & $\begin{array}{c}<60 \mathrm{mK} \\
(\mathrm{F} / 1,300 \mathrm{~K}, \\
60 \mathrm{~Hz})\end{array}$ & $\begin{array}{c}<75 \mathrm{mK} \\
(\mathrm{F} / 1,300 \mathrm{~K} \\
30 \mathrm{~Hz})\end{array}$ \\
\hline resolution & $640 \times 480$ & $640 \times 480$ \\
\hline pixel pitch & $25 \mu \mathrm{m}$ & $17 \mu \mathrm{m}$ \\
\hline $\begin{array}{c}\text { power } \\
\text { consumption }\end{array}$ & $<300 \mathrm{~mW}$ & $<170 \mathrm{~mW}$ \\
\hline
\end{tabular}

proportional voltage signals and temperature variations inside and outside the cameras. 
Ambient temperature was supplied by a climate chamber. Temperature started at $25^{\circ} \mathrm{C}$, was raised to $35^{\circ} \mathrm{C}$ and after reaching the steady state decreased back to $25^{\circ} \mathrm{C}$. During the complete process both cameras were looking at four radiation sources with different temperatures. For better comparability, infrared images of both cameras were already preprocessed including bad pixel replacement and two-point non-uniformity correction. Figure 1 shows the spatial mean values of the TEC-less measurement.

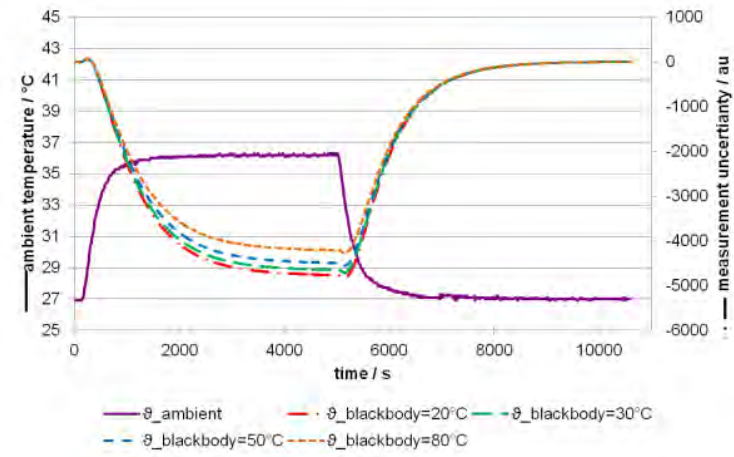

Fig. 1: Influence of ambient temperature on voltage signal of a TEC-less sensor.

The measured voltage signal of the infrared camera with stabilized constant sensor temperature due to an increasing internal temperature increases for each radiation source. For the TEC-less sensor two effects superimpose. An increasing signal derived from the increasing internal temperature and the decreasing signal due to the warming of the sensor. The decreasing measurement signal is caused by a negative thermal coefficient of resistance (TCR) of the resistor material used in the microbolometer. However, the effect caused by heating the sensor is dominant. It should be noticed that the higher the object temperature is the lower is the measurement deviation.

During measurement two temperature probes were detecting the internal camera temperature, one close to the sensor and a second one in the camera housing. Together with an integrated thermal diode and an external thermocouple inside the climate chamber this enabled us to record temperature variations in the sensor and in the camera (Fig. 2). It has to be mentioned that power consumption rose due to higher temperatures, about $3 \%$ with TEC and $0.3 \%$ without TEC, leading to an additional temperature gain.
Camera temperature and ambient temperature are equal in the steady state; remaining differences are caused by measurement uncertainties of both probes. The relation between ambient and internal camera temperature shows low pass behavior. The temperature gradient and its variation in time are the same for both cases with and without TEC. In the steady state the temperature difference between sensor case and the camera interior is independent of ambient temperature and almost to about $10 \mathrm{~K}$. The low pass behavior between the temperatures of the camera interior and the focal plane array (FPA) causes the response delay shown in figure 1 .

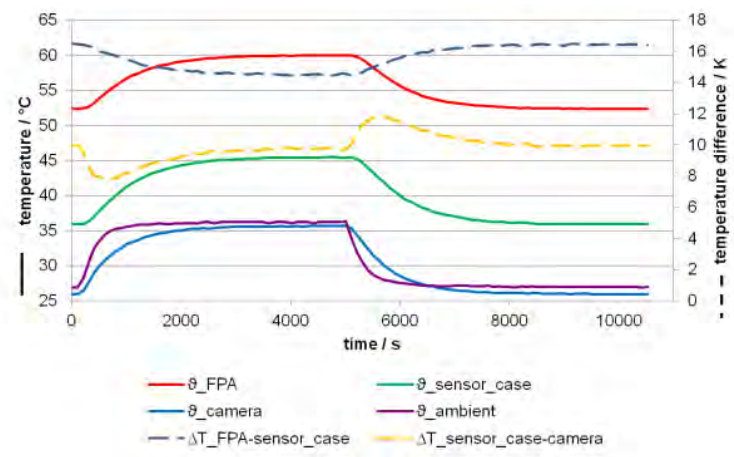

Fig. 2: Measured temperatures and temperature gradients in variation of time of the TEC-less infrared camera.

\section{Sensor parameters}

In a next step, the influence of external conditions on the radiation measurement with the camera shall be studied. To do this a simplified set-up will be considered which compromises only the sensor and the radiation source as main components (Fig. 3). A thick copper plate with a coating of high emissivity is used as blackbody. Peltier elements are used to control the temperatures of both the blackbody and the detector. We assume that all sensor pixels get almost the same amount of radiation from the blackbody radiator. The estimated difference in incoming radiation between pixels in the middle of the sensor array and at the corner is about $0.22 \%$. We used the same kind of TEC-less detector such as in the previous experiment (section 2) [3]. The set-up of figure 3 was used to determine the crucial sensor parameters describing the properties of FPA sensor pixels. 


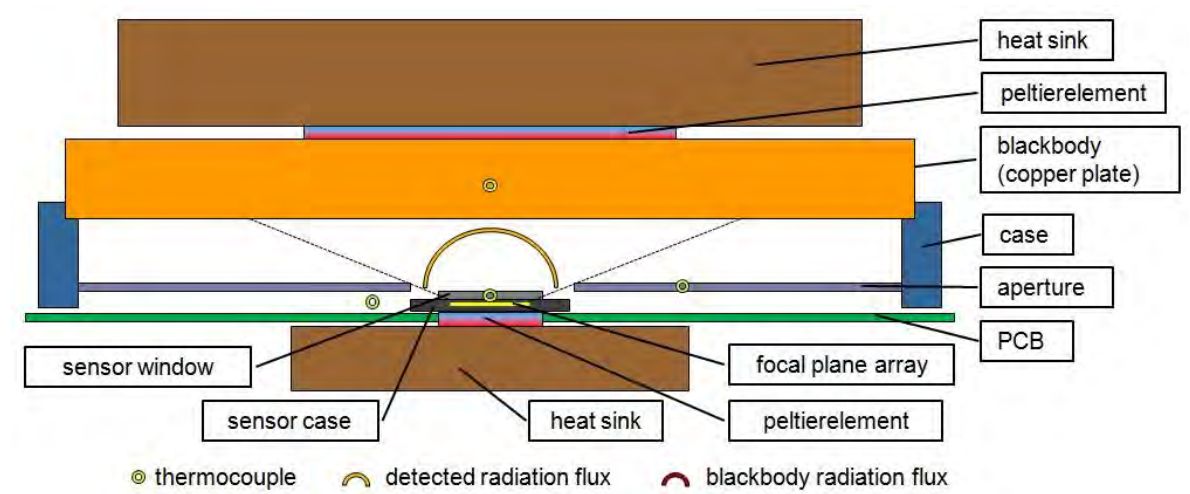

Fig. 3: Simplified radiation measurement set up for sensor characterization.

\section{a) Non-uniformity}

Different sensor pixels respond to the incident radiation with different characteristic curves due to tolerances in geometry and fabrication. To correct these pixel non-uniformities a reference curve (3.1) is used, where $V$ is the reference sensor voltage, $T_{O}$ the object temperature and gain norm $_{\text {and }}$ offset $t_{\text {norm }}$ the curve parameters of this so-called normal curve. Each pixel voltage $V_{i j}$ with its individual characteristic curve (3.2) can now be corrected by a two-point correction (3.3) [1].

$$
\begin{gathered}
V\left(T_{O}\right)=\text { gain }_{\text {norm }} \cdot T_{O}+\text { offset }_{\text {norm }} \\
V_{i j}\left(T_{O}\right)=\text { gain }_{i j} \cdot T_{O}+\text { offset }_{i j} \\
V_{S, i j}^{\prime}\left(T_{O}\right)=\frac{\text { gain }_{\text {norm }}}{\text { gain }_{i j}} \cdot V_{S, i j}\left(T_{O}\right)+o f f \text { set }_{\text {norm }}-\frac{\text { gain }_{\text {norm }}}{\text { gain }_{i j}} \cdot \text { offset }_{i j}
\end{gathered}
$$

\section{b) Temperature dependency}

The resistor material of the bolometer detector array used here consists of amorphous silicon. It has a negative thermal coefficient of resistance (TCR). Due to the TCR, gain norm $_{\text {and }}$ offset $_{\text {norm }}$ depend both on the FPA's temperature (Fig. 4). The TCR has a huge influence on signal responds of the sensor as mentioned in the thermal drift experiment above.

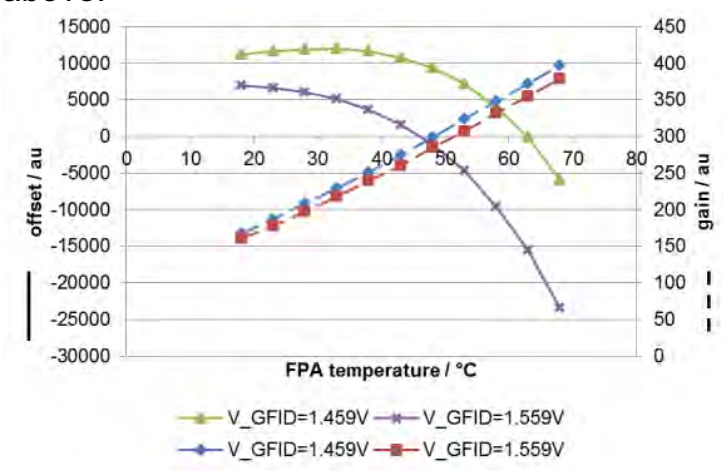

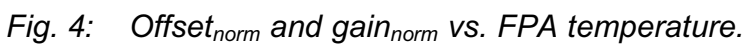
Parameter $V_{G F I D}$ controls operation point.

\section{c) Pixel field of view}

The radiation fluxes coming from the scene and from the interior of the camera we have to be weighted depending on the angle of incidence and the position of the detecting sensor element. Therefore, it is essential to determine the field of view of each pixel. This was done by rotating a small radiation source equidistantly around the detector. The surface of the radiation source had a size of about $300 \mathrm{~mm}^{2}$ and mean surface temperature of $130^{\circ} \mathrm{C}$. Figure 5 shows the detection characteristic of several pixels. One may notice that pixel field of view and direction of highest incident radiation depends on the pixel position. 


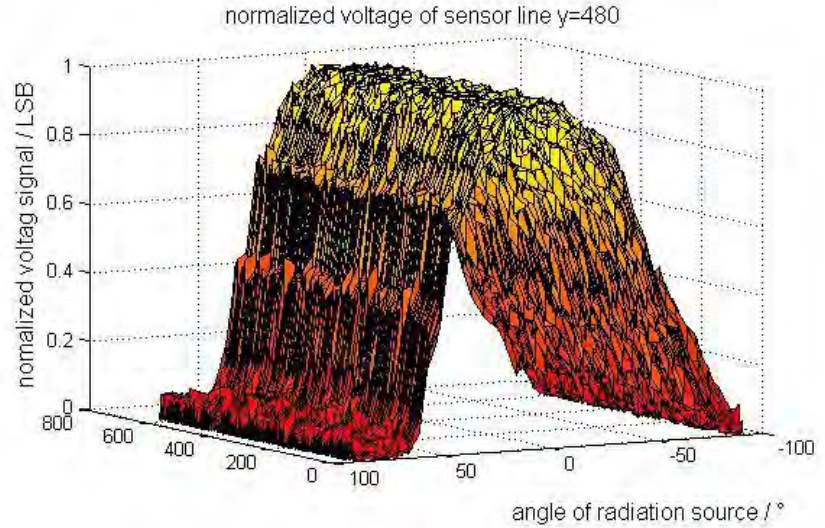

(a)

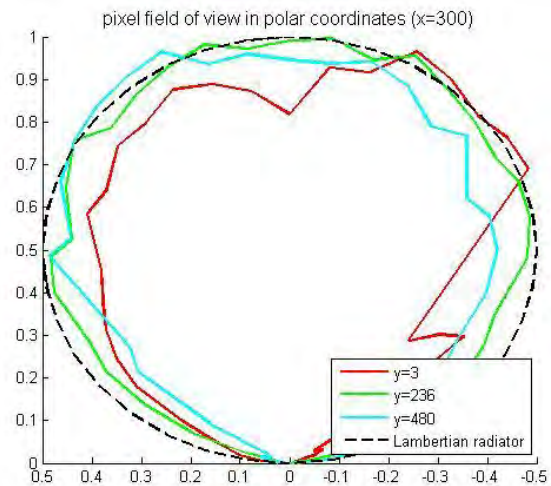

(b)

Fig. 5: Pixel field of view function of sensor line 480 (a). Viewing characteristic in polar coordinates of pixels (300,3), $(300,236)$ and $(300,480)(b)$.

\section{d) Transmittance of sensor window}

The sensor window enables the incident radiation to hit the sensor pixels and caps the vacuum around the FPA. Usually, Si windows with antireflective coating are used for the sensor window. The transmittance in the IR range up to $10 \mu \mathrm{m}$ amounts to ca. 0.9. This values decreases with higher wavelengths [4].

\section{$4 \quad$ Camera model}

Figure 6 shows a simple scheme of an infrared camera. In order to develop a generic model of the radiation detection process all temperature influences mentioned above have to be considered and included.

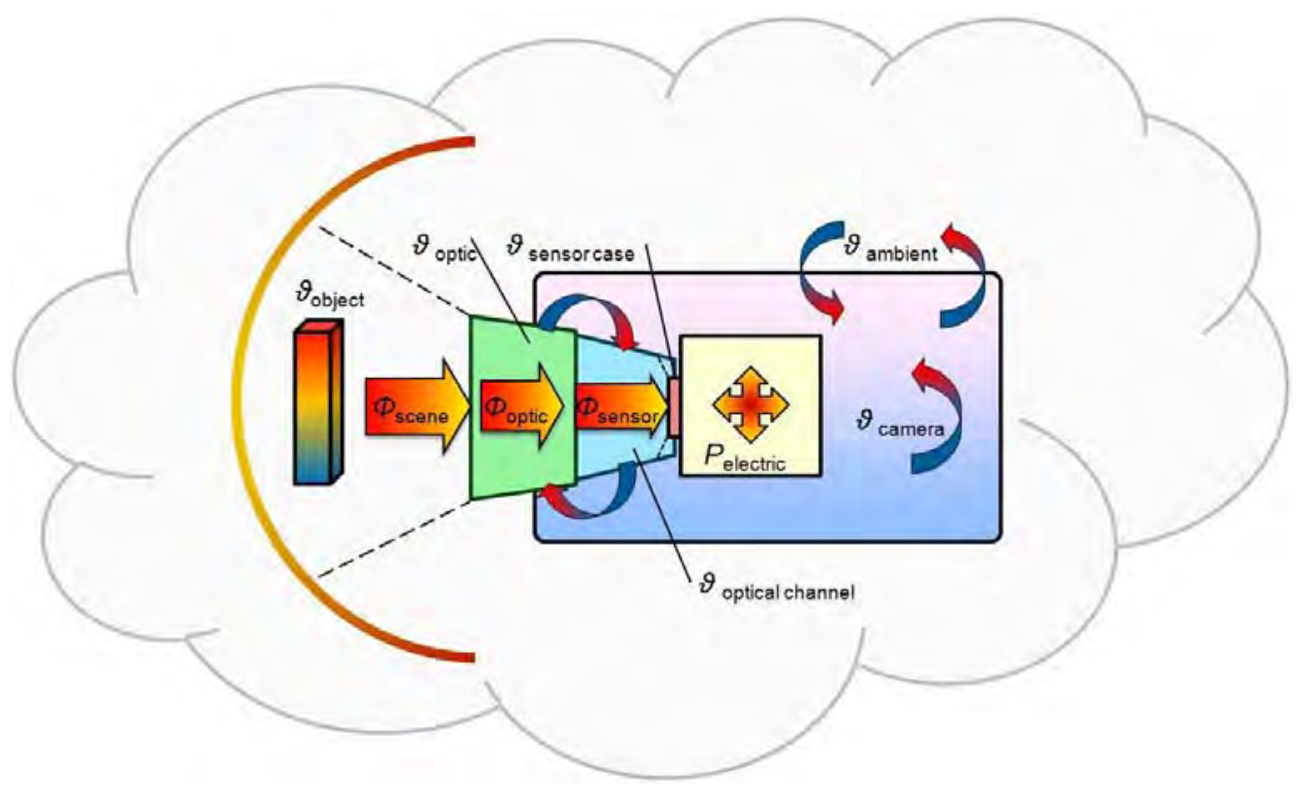

Fig. 6: Camera model with radiation paths and relevant temperatures influencing the measurement signal.

In a first step, the basic measurement set-up was extended by including measures to supply defined thermal conditions (Fig. 7). The set-up compromises a one-sided heatable aperture with an f-number $F=1$. This allows generating a temperature gradient and studying its influence on radiation measurement. Additional thermocouples monitor the temperature distribution on the surface of the aperture. 


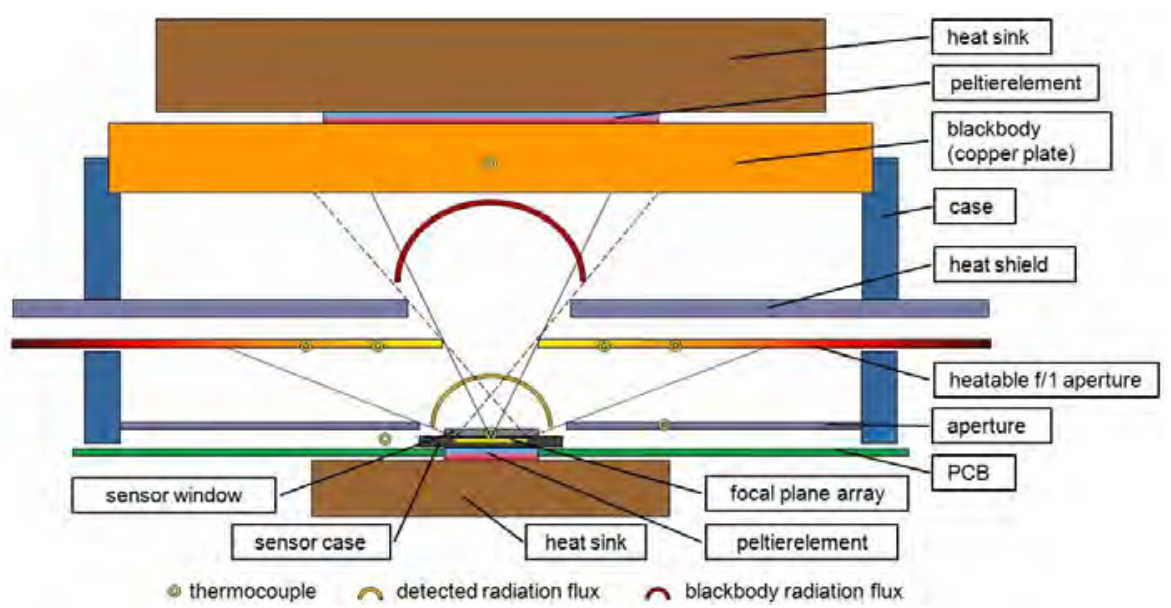

Fig. 7: Measurement set-up including a heatable F/1 aperture.

Due to the aperture geometry and depending on the pixel position, the projected solid angles $\omega_{\text {blackbody, }, j}$ and $\omega_{\text {aperture, } i j}$ differ for each pixel in relation to the blackbody radiation $L_{\text {blackbody }}$ and the radiation $L_{\text {aperture }}$ from the aperture, respectively. The radiation $L_{\text {aperture }}$ from the aperture is defined by the temperature gradient.
Each pixel emits radiation depending on the FPA temperature. The radiant exitance $L_{\text {pixel }}$ is constant for each pixel and is directed into the complete half space $\left(\omega_{\text {halfspace }}=\pi\right)$. The irradiance $E_{i j}$ of each pixel can be calculated from these quantities as:

$$
E_{i j}=L_{\text {blackbody }} \cdot \omega_{\text {blackbody }, i j}+\int_{\omega_{\text {aperture }, j}} L_{\text {aperture }}(\omega) d \omega-L_{\text {pixel }} \cdot \omega_{\text {halsspace }} .
$$

In the first experiments using the extended set-up we measured the influence of supplied aperture temperature gradients on the radiation measurement in order to verify our calculations. The blackbody temperature and the FPA temperature remained constant. Figure 8 the compares the measured radiation-proportional voltage signal $V_{i j}$ with the calculated irradiance $E_{i j}$ of each pixel at a give aperture temperature gradient. Normalizing both values allows us to compare them directly. It can be seen that both curves agree sufficiently.

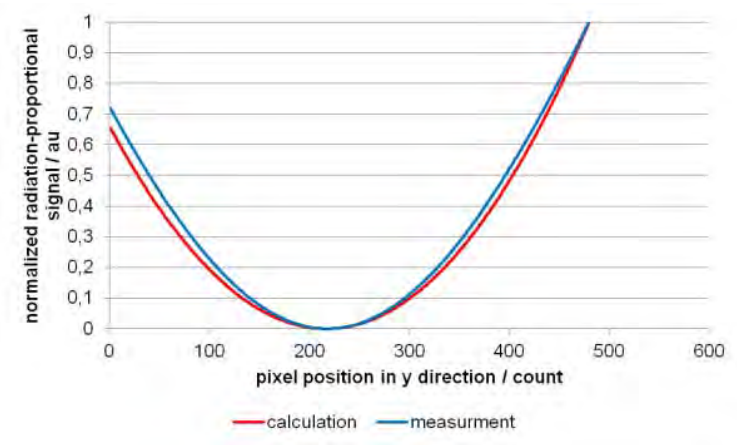

Fig. 8: Normalized radiation-proportional signal of measurement and calculation vs. pixel position.

\section{$5 \quad$ Further researches}

The uncertainty between measurement and calculation will be studied. The measured radiation signal will be corrected by subtracting the calculated radiation from the aperture under consideration of its temperature gradient. This correction will be analyzed in the steady and the transient state.

\section{Acknowledgements}

This work was financially supported by the Deutsche Forschungsgemeinschaft Germany under contract GE779/26-1.

\section{References}

[1] H. Budzier, G. Gerlach: Thermal Infrared Sensors; Wiley 2011

[2] http://www.ulis-ir.com/uploads/Products/Pico640E-UL04322_v2.pdf

[3] http://www.ulis-ir.com/uploads/Products/Pico640P-UL04272_v2.pdf

[4] http://www.optotl.com/prod/prosv/Si - 5) № ARSI-8-14 